\title{
One-Year Late Lumen Loss between A Polymer-Coated Paclitaxel- Eluting Stent (Eluvia) and a Polymer-Free Paclitaxel-Coated Stent (Zilver PTX) for Femoropopliteal Disease
}

\author{
Yoshimitsu Soga ${ }^{1}$, Masahiko Fujihara ${ }^{2}$, Yusuke Tomoi ${ }^{1}$, Osamu lida ${ }^{3}$, Takayuki Ishihara ${ }^{3}$, Daizo Kawasaki ${ }^{4}$ \\ and Kenji Ando ${ }^{1}$
}

${ }^{1}$ Kokura Memorial Hospital, Department of Cardiology, Kitakyushu Japan

${ }^{2}$ Kishiwada Tokushukai Hospital, Department of Cardiology, Kishiwada, Japan

${ }^{3}$ Kansai Rosai Hospital, Cardiovascular center, Amagasaki, Japan

${ }^{4}$ Morinomiya Hospital, Department of Cardiology, Osaka, Japan

Aim: Paclitaxel-eluting stents' (Eluvia and Zilver PTX) effectiveness has been recently reported for femoropopliteeal (FP) lesions. However, there is no evaluation of one-year late lumen loss (LLL). Therefore, we evaluated one-year LLL after implantation with Eluvia or Zilver PTX.

Methods: This was a multicenter, prospective study. Patients who had symptomatic de novo lesions in the native FP artery were enrolled. The primary endpoint was one-year angiographic LLL, and the secondary endpoints were binary restenosis and target lesion revascularization (TLR) at one year.

Results: From December 2015 to December 2016, 48 patients (Eluvia, 36 patients; Zilver PTX, 12 patients) were enrolled. No significant difference was found in baseline and lesion characteristics between both groups. One-year, LLL was significantly lower in the Eluvia group $(0.60$ \{plus minus\} $0.80 \mathrm{~mm})$ than in the Zilver PTX group $(1.74$ \{plus minus\} $0.89 \mathrm{~mm})(P=0.0003)$. Negative LLL was observed only in the Eluvia group $(0 \%$ vs. $23 \%, p=0.096)$. The binary restenosis rate was significantly lower than in the Zilver PTX group ( $0 \%$ vs. $16.7 \%$, $P=0.012)$. The one-year TLR in the Eluvia group tended to be lower $(0 \%$ vs. $8.3 \%, P=0.08)$. Stent thrombosis was not observed in either group.

Conclusion: One-year LLL in the Eluvia group was significantly lower than that in the Zilver PTX group for FP lesions.

Key words: Drug-eluting stent, Femoropopliteal disease, Endovascular therapy

\section{Introduction}

To date, endovascular therapy (EVT), combined with a nitinol stent, as the revascularization method for peripheral artery disease (PAD) with femoropopliteal (FP) lesions has shown better short- and mediumterm results compared to using only a balloon ${ }^{1-6)}$. However, the problem of restenosis occurring during the chronic phase has not yet been resolved. With the introduction of the drug-coated balloon (DCB), the rates of both restenosis and target lesion revasculariza- tion (TLR) have decreased ${ }^{7,8)}$. However, successful initial vessel preparation using standard PTA is necessary in order to proceed with treatment using a DCB; therefore, it difficult to make DCBs the first-choice treatment. In recent years, a polymer-coated paclitaxel-eluting stent (Eluvia) has become available, and revascularization strategies for FP lesions are changing. In the MAJESTIC trial ${ }^{9)}$, an international, multicenter single-arm study, the primary patency in the first year was $96.4 \%$ and in the second year was $83.5 \%$, which are very high patency rates. The IMPE-

Address for correspondence: Yoshimitsu Soga, Department of Cardiology, Kokura Memorial Hospital, 3-2-1 Asano, Kokurakita-ku, Kitakyushu, Japan. 802-0001 E-mail: soga@circulation.jp

Received: April 10, 2019 Accepted for publication: May 21, 2019

Copyright@2019 Japan Atherosclerosis Society

This article is distributed under the terms of the latest version of CC BY-NC-SA defined by the Creative Commons Attribution License. 
RIAL trial ${ }^{10)}$, which compared a polymer-coated paclitaxel-eluting stent (Eluvia) to a polymer-free paclitaxel-coated stent (Zilver PTX), revealed that, for primary patency, the Eluvia stent achieved significantly better results than the Zilver PTX stent. However, evaluation of late lumen loss (LLL) findings after stenting has not been reported. Here, we evaluated the one-year LLL for FP lesions implanted with either an Eluvia stent or a Zilver PTX stent.

\section{Methods}

\section{Study Design}

This was a multicenter, prospective, clinical study in four Japanese institutions between December 2015 and December 2016. Patients eligible for the study were at least 40 years old and had chronic, symptomatic, lower limb ischemia, defined as Rutherford category 2, 3, or 4, and de novo stenotic or occlusive lesions in the native FP artery with at least one below-the-knee runoff patent to the ankle. The vessel diameter was to be $\geq 4 \mathrm{~mm}$ and $\leq 6 \mathrm{~mm}$, and the total lesion length was $\geq 30 \mathrm{~mm}$ and $\leq 200 \mathrm{~mm}$.

Exclusion criteria were restenosis lesion, renal failure (serum creatinine $>2 \mathrm{mg} / \mathrm{dL}$ ) or dialysis, acute onset ischemia and/or acute thrombosis, presence of aneurysm in the target vessel, use of debulking devices in the target vessel, history of major amputation in the target limb, prior surgery in the target limb, history of myocardial infarction (MI) or stroke/cerebrovascular disease (CVD) within six months, unstable angina pectoris, Type 1 diabetes, history of bleeding diathesis, documented life expectancy $<2$ years, known hypersensitivity or contraindication to contrast media, known hypersensitivity/allergy to the investigational stent system or protocol related therapies (e.g., nitinol, paclitaxel, or structurally related compounds, polymer or individual components, and antiplatelet, anticoagulant, thrombolytic medications), pregnant, breast feeding, or planning to become pregnant. The study population was included in the IMPERIAL trial. Yoshimitsu Soga, Masahiko Fujihara, Osamu Iida, and Daizo Kawasaki received honoraria (e.g. lecture fee) from the both Boston Scientific Japan and Cook Medical.

After successful implantation of either Eluvia or Zilver PTX, the need for follow-up angiography was comprehensively explained prior to discharge, and patients from whom written consent could be obtained were enrolled. In principle, follow-up angiography was performed after six months \pm 1 month (if possible) and 12 months \pm 1 month. For follow-up angiography, the same site was imaged using a dedicated ruler for quantitative vascular analysis (QVA) in the same direction. An independent core lab measured QVA (lesion length, reference vessel diameter, \% diameter stenosis, minimum lumen diameter [MLD], LLL) and peripheral arterial calcium scoring system (PACSS) grade ${ }^{11)}$ with CAASV software (version 5.9.2; Pie Medical Imaging, Maastricht, the Netherlands).

The study protocol was approved by each hospital's ethics committees, and the study was performed in accordance with the Declaration of Helsinki. Every patient provided written informed consent.

\section{Study Outcome Measures}

The primary outcome measure was 12-month angiographic LLL. The secondary outcome measures were binary restenosis and clinically-driven target lesion revascularization (CD-TLR) at 12 months.

\section{Procedures and Follow-Up}

All patients were medicated with dual antiplatelet therapy (Aspirin 100mg/day + clopidogrel $75 \mathrm{mg} /$ day) before the procedure. After insertion of a $6 \mathrm{Fr}$ sheath, an intra-arterial bolus of 5000 IU of heparin was injected and supplemented, as required, to maintain an active clotting time of $>200$ s. A $0.035-$, 0.018 - or 0.014 -inch guidewire was used to cross the lesion. After passing the wire, balloon angioplasty was performed. All lesions were dilated with an optimally sized balloon. Two types of drug-eluting stents were used: Eluvia (Boston Scientific, Marlborough, MA, USA) and Zilver PTX Stent System (Cook Medical, Bloomington, IN, USA). The stent size was chosen to be $1 \mathrm{~mm}$ larger than the reference vessel diameter. The lesion had to be fully covered with Eluvia or Zilver PTX.

After the procedure, all patients were prescribed lifelong aspirin $(100 \mathrm{mg} /$ day), and prolonged (at least three months) clopidogrel $75 \mathrm{mg} /$ day was recommended. The presence of stent fracture, and duplex ultrasound of the stented vessel, were monitored at 1 , 6, 12 months.

\section{Definitions}

Restenosis was defined as $>50 \%$ of diameter stenosis by angiography. Clinically-driven TLR was defined as re-intervention performed for greater than $50 \%$ diameter stenosis within $5 \mathrm{~mm}$ of the target lesion, identified by angiography after documentation of recurrent clinical symptoms. The LLL was defined as the difference between post-procedural and followup MLD $^{12)}$. Negative LLL was defined as a negative LLL value. Stent fracture was defined as clear interruption of stent struts, identified by X-ray from more than two projections, with resulting kink or misalign- 


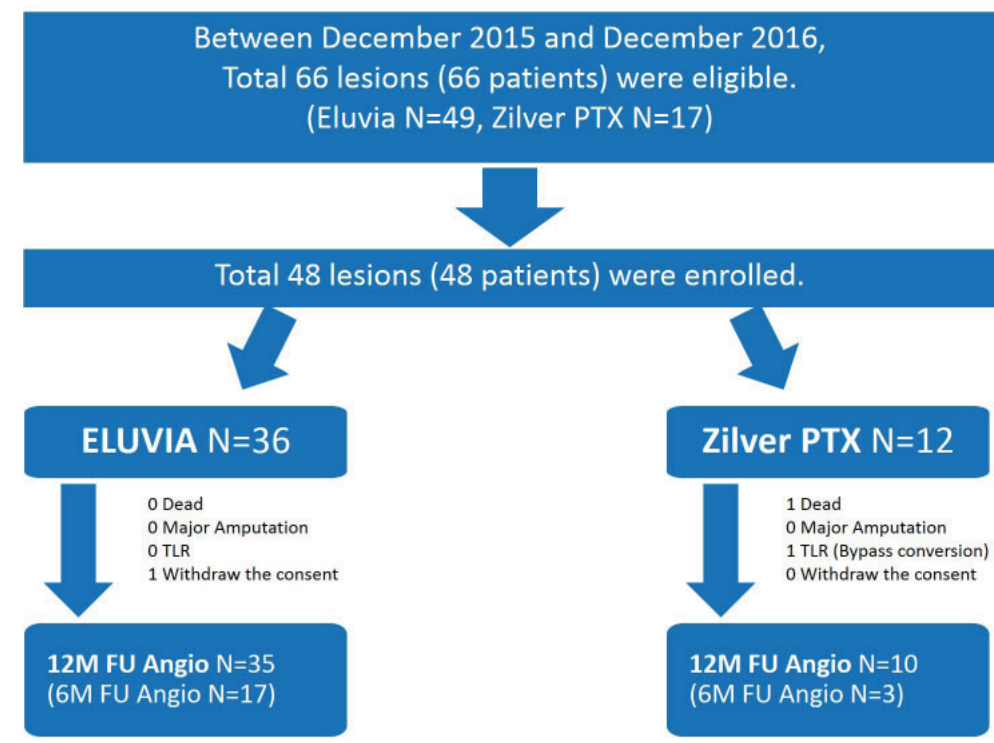

Fig. 1. Participant Flow

ment along the axial length of the stent. Coronary artery disease (CAD) was defined as stable angina with documented CAD, history of percutaneous coronary intervention, history of coronary artery bypass graft surgery, or previous MI. Cerebrovascular disease (CVD) was defined as a hospital or neurologist's report with a diagnosis of transient ischemic attack or ischemic stroke.

\section{Statistical Analysis}

Continuous variables were expressed as mean \pm SD. Differences among treatment groups were evaluated by analysis of variance for continuous variables and by the chi-square or Fisher exact test for categorical variables. A probability value of $<0.05$ was considered statistically significant.

\section{Results}

\section{Baseline}

Between December 2015 and December 2016, 66 patients (Eluvia group, 49 patients; Zilver PTX group, 17 patients) were considered eligible for the study; 48 patients (Eluvia group, 36 patients; Zilver PTX group, 12 patients) consented to a follow-up study after one year (Fig. 1). Patient characteristics are summarized in Table 1. No significant difference was found in terms of patient characteristics between both groups.

Of the 48 patients (48 lesions) who provided consent for follow-up contrast radiography after one year, one patient died due to a non-cardiovascular disease (Zilver PTX group), one patient withdrew con- sent (Eluvia group), and one patient underwent bypass surgery (Zilver PTX group) due to restenosis; thus, follow-up imaging could be performed one year later in 45 patients (Eluvia group, 35 patients; Zilver PTX group, 10 patients) (Fig. 1).

\section{Outcome Measures (Fig. 2)}

The binary restenosis rate at one year was significantly lower in the Eluvia group, at $0 \%(0 / 36)$, than it was in the Zilver PTX group, at $16.7 \%(2 / 12)(P=$ $0.012)$. The one-year TLR was $0 \%(0 / 36)$ for the Eluvia group and $8.3 \%(1 / 12)$ for the Zilver PTX group $(P=0.08)$. Further, two cases of stent fracture were noted in the Eluvia group $(5.9 \%)$ but none $(0 \%)$ in the Zilver PTX group $(P=0.46)$. Notably, stent thrombosis was not observed in either group.

\section{Quantitative Vascular Analysis (QVA)}

No difference was noted in terms of preoperative and postoperative QVA results between the two groups. In the examination of 17 cases of the Eluvia group and 3 of the Zilver PTX group, in which follow-up angiography could be performed six months later (Table 2), MLD was significantly larger in the Eluvia group $(4.2 \pm 0.9 \mathrm{~mm})$ than in the Zilver PTX group $(3.2 \pm 0.5 \mathrm{~mm})(P=0.07)$. However, LLL was significantly lower in the Eluvia group $(0.12 \pm 0.6$ $\mathrm{mm})$ than in the Zilver PTX group $(1.53 \pm 1.1 \mathrm{~mm})$ $(P=0.009)$. We comparatively examined 35 cases of the Eluvia group and 10 of the Zilver PTX group in which follow-up angiography could be performed one year later (Table 2). After 12 months, MLD was still significantly larger in the Eluvia group $(4.2 \pm 1.0 \mathrm{~mm})$ 
Table 1. Baseline Characteristics

\begin{tabular}{|c|c|c|c|}
\hline & Eluvia $(N=36)$ & Zilver PTX $(N=12)$ & $P$ value \\
\hline Age (yrs) & $73 \pm 9$ & $76 \pm 5$ & 0.27 \\
\hline Male (\%) & $30(83)$ & $8(67)$ & 0.22 \\
\hline Body mass index & $22.5 \pm 3.6$ & $24.5 \pm 4.3$ & 0.13 \\
\hline Current smoker (\%) & $11(31)$ & $2(17)$ & 0.35 \\
\hline Hypertension (\%) & $34(94)$ & $12(100)$ & 0.40 \\
\hline Dyslipidemia (\%) & $25(69)$ & $8(67)$ & 0.86 \\
\hline Diabetes (\%) & $20(56)$ & $7(58)$ & 0.87 \\
\hline Coronary artery disease (\%) & $23(64)$ & $8(67)$ & 0.86 \\
\hline Cerebrovascular disease (\%) & $8(22)$ & $4(33)$ & 0.44 \\
\hline Heart failure (\%) & $4(11)$ & $3(25)$ & 0.24 \\
\hline Atrial fibrillation (\%) & 7 (19) & $0(0)$ & 0.10 \\
\hline Pre-procedural ABI & $0.69 \pm 0.15$ & $0.72 \pm 0.13$ & 0.55 \\
\hline Post-procedural ABI & $0.95 \pm 0.11$ & $0.98 \pm 0.14$ & 0.47 \\
\hline \multicolumn{4}{|l|}{ Rutherford Category } \\
\hline $2 / 3 / 4$ & $21 / 15 / 0$ & $6 / 5 / 1$ & 0.21 \\
\hline \multicolumn{4}{|l|}{ Medication } \\
\hline Aspirin (\%) & $36(100)$ & $12(100)$ & 1.00 \\
\hline P2Y12 inhibitor (\%) & $36(100)$ & $12(100)$ & 1.00 \\
\hline Statin (\%) & $21(58)$ & $9(75)$ & 0.30 \\
\hline Anticoaglant (\%) & 7 (19) & $0(0)$ & 0.10 \\
\hline \multicolumn{4}{|l|}{ Lesion and Procedure } \\
\hline \multicolumn{4}{|l|}{ TASC classification } \\
\hline $\mathrm{A} / \mathrm{B} / \mathrm{C} / \mathrm{D}$ & $11 / 14 / 10 / 1$ & $3 / 9 / 0 / 0$ & 0.10 \\
\hline Lesion Length (mm) & $116 \pm 52$ & $97 \pm 30$ & 0.24 \\
\hline Reference diameter (mm) & $5.6 \pm 1.1$ & $6.1 \pm 1.2$ & 0.20 \\
\hline Chronic total occlusion (\%) & $6(17)$ & $1(8)$ & 0.48 \\
\hline \multicolumn{4}{|l|}{ PACSS grade (\%) } \\
\hline $0 / 1 / 2 / 3 / 4$ & $15 / 11 / 3 / 3 / 4$ & $5 / 1 / 3 / 1 / 2$ & 0.41 \\
\hline Stent Diameter (mm) & $6.54 \pm 0.48$ & $6.63 \pm 0.48$ & 0.61 \\
\hline IVUS usage (\%) & $29(81)$ & $9(75)$ & 0.68 \\
\hline
\end{tabular}

PACSS: peripheral arterial calcium scoring system IVUS; intravascular ultrasound

Late Lumen Loss

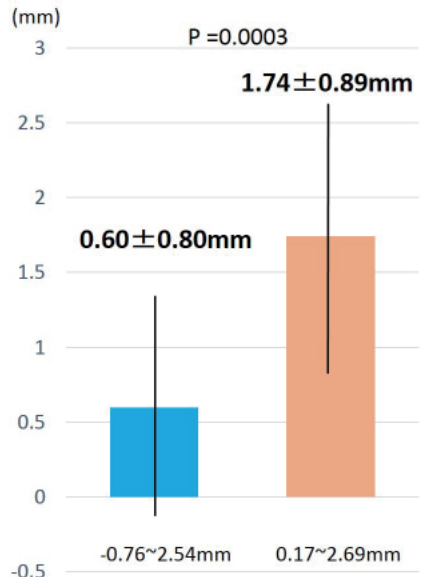

Eluvia
Restenosis

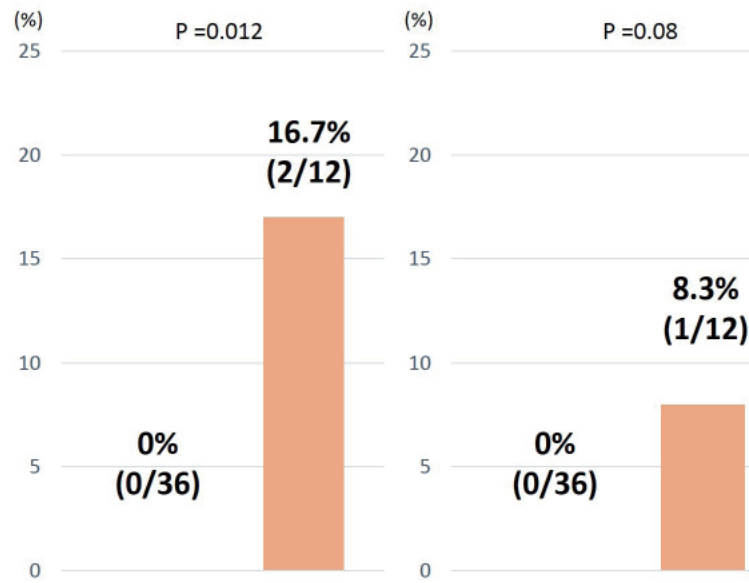

Fig. 2. Clinical Outcome Measures Eluvia Zilver PTX TLR; target lesion revascularization 
Table 2. Quantitative vascular analysis

\begin{tabular}{|c|c|c|c|}
\hline & Eluvia $(N=35)$ & Zilver PTX $(N=10)$ & $P$ value \\
\hline \multicolumn{4}{|l|}{ Pre-procedure } \\
\hline Lesion Length (mm) & $96.6 \pm 50.8$ & $92.0 \pm 55.4$ & 0.80 \\
\hline Reference vessel diameter (mm) & $4.6 \pm 0.6$ & $4.5 \pm 0.8$ & 0.75 \\
\hline \% Diameter Stenosis (\%) & $74.7 \pm 17.7$ & $67.2 \pm 21.6$ & 0.26 \\
\hline Minimum lumen diameter (mm) & $1.0 \pm 0.7$ & $1.3 \pm 08$ & 0.31 \\
\hline \multicolumn{4}{|l|}{ Post-procedure } \\
\hline \% Diameter Stenosis (\%) & $17.0 \pm 8.1$ & $15.6 \pm 6.48$ & 0.63 \\
\hline Minimum lumen diameter $(\mathrm{mm})$ & $4.7 \pm 0.7$ & $4.9 \pm 06$ & 0.50 \\
\hline 6-Month Follow-Up & $(N=17)$ & $(N=3)$ & \\
\hline \% Diameter Stenosis (\%) & $21.0 \pm 12$ & $24.0 \pm 22$ & 0.73 \\
\hline Minimum lumen diameter $(\mathrm{mm})$ & $4.2 \pm 0.9$ & $3.2 \pm 05$ & 0.07 \\
\hline Late Lumen Loss (mm) & $0.12 \pm 0.6$ & $1.53 \pm 1.1$ & 0.009 \\
\hline 12-Month Follow-Up & $(N=35)$ & $(N=10)$ & \\
\hline \% Diameter Stenosis (\%) & $26.4 \pm 12.0$ & $32.3 \pm 11.4$ & 0.17 \\
\hline Minimum lumen diameter $(\mathrm{mm})$ & $4.2 \pm 1.0$ & $3.2 \pm 06$ & 0.005 \\
\hline Late Lumen Loss (mm) & $0.6 \pm 0.8$ & $1.7 \pm 0.9$ & 0.000 \\
\hline
\end{tabular}

than in the Zilver PTX group $(3.2 \pm 0.6 \mathrm{~mm})(P=$ $0.0047)$, and LLL was significantly lower in the Eluvia group $(0.60 \pm 0.80 \mathrm{~mm})$ than in the Zilver PTX group $(1.74 \pm 0.89 \mathrm{~mm})(P=0.0003)$. A comparison of contrast-enhanced imaging results of the lower extremities in typical cases after one year revealed a difference in terms of neointimal proliferation. No negative LLL after one year was observed in the Zilver PTX group, but it was observed in $23 \%$ (8 of 35) of the Eluvia group ( $0 \%$ vs. $23 \%, p=0.096$; [0.03 to $-0.76 \mathrm{~mm}]$ ). Serial angiographic follow-up cases are shown in Fig. 3. In the Eluvia group, LLL was very low in each. Representative negative LLL is shown in Fig.4. Instent vessel lumen enlargement was found after six months on a follow-up angiogram. It was maintained up to one year. In patients with IVUS findings, lumen narrowing was confirmed in the Zilver PTX group, but there was almost no narrowing in the Eluvia group at one year; the difference in terms of LLL was visually identified as well (Fig.5). To confirm the neointimal coverage of Eluvia stent at one year, an angioscope was performed. Angioscopic findings confirmed neointimal coverage of stent struts without thrombus adhesion (Fig. 6). All patients were followed up and duplex ultrasound was performed, but aneurysmal degeneration was not observed.

\section{Discussion}

In this study, we performed follow-up angiography during chronic phases in the patients of the Eluvia and Zilver PTX groups and compared LLL one year later. The Eluvia group had significantly lower
LLL than the Zilver PTX group. During the BNS era, the peak of restenosis was one year ${ }^{13)}$. In this study, the one-year restenosis rate of the Eluvia group was extremely low (0\%). This is a very different result compared to the Innova stent as platform ${ }^{14)}$. Thus, whether there is a peak of restenosis for Eluvia should be evaluated using accumulated cases and long-term follow-ups.

In this study, negative LLL was observed in 23\% patients (8 of 35) of the Eluvia group, wherein the vessel diameter during the chronic phase was larger than that at the final imaging. To investigate previously reported information on aneurysmal degeneration $^{15)}$, we performed duplex ultrasound for all cases, but aneurysmal degeneration was not observed in the present study. Similarly, for angiographic follow-up, there was no aneurysmal change exceeding the stent diameter. Some patients had a large negative LLL (Supplement D). The vessel diameter along the stent clearly indicated an enlargement compared with the native vessel on the contralateral side, but aneurysm formation was not observed. Further evaluation is warranted to determine whether such enlargement affects events occurring during the chronic phase.

In this study, although stent thrombosis was not noted in either group, the duration of dual antiplatelet therapy (DAPT) was determined to be at least three months, but it continued in all patients for one year, except in cases involving temporary withdrawal due to dental or surgical treatment. An important factor in the discussion of the possibility of shortening of the DAPT period and optimal DAPT duration is the time when a stent strut is completely covered. If restenosis 

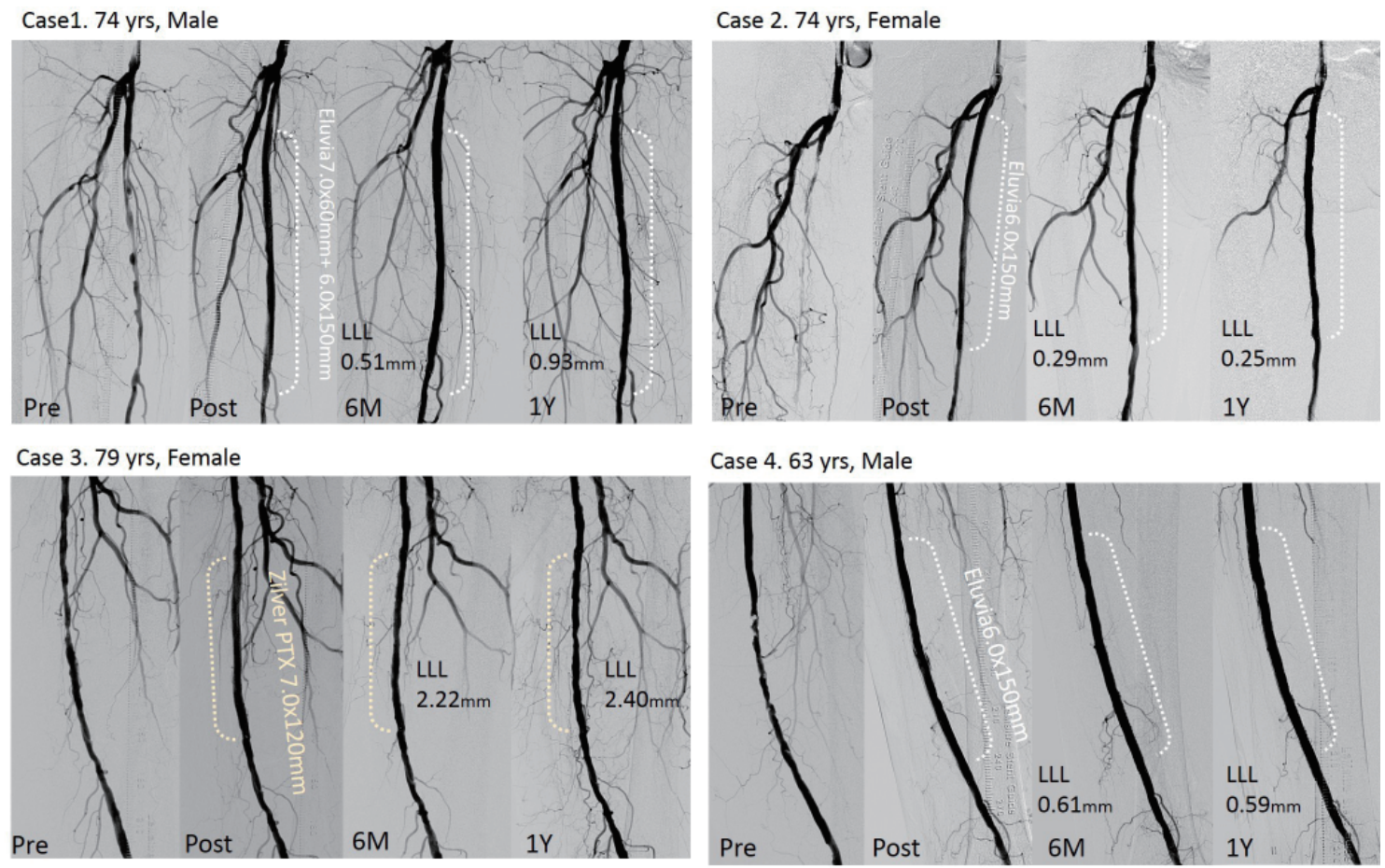

Fig. 3. Representative cases of Serial Angiographic Follow-Up

LLL; late lumen loss

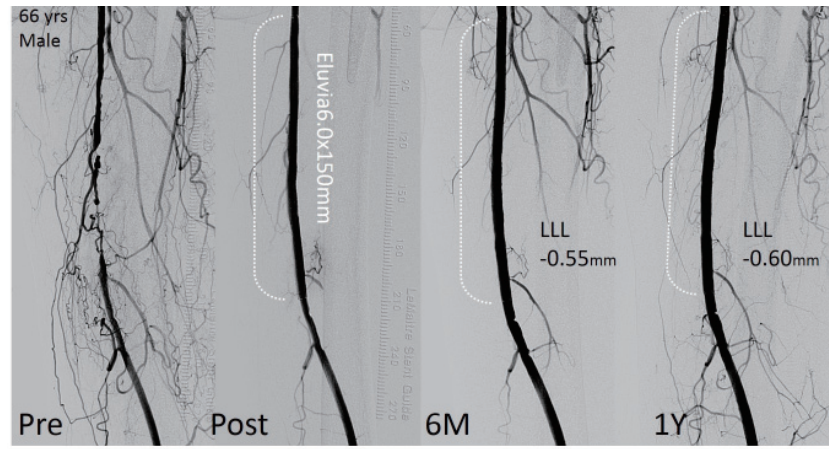

Fig.4. Representative case of Negative Late Lumen Loss LLL; late lumen loss

suppression is accompanied by delayed endothelialization, a longer DAPT duration may be desirable owing to the fear of induction of stent thrombosis. However, another concern is careless DAPT continuation due to increased hemorrhagic events, and it is necessary to examine an accurate method for stent coverage evaluation. Nevertheless, in the Zilver PTX group, even when the stent was covered, partial necrotic tissue was noted, rather than a neointima ${ }^{16}$, and the issue was determining how long the stent strut would take for complete coverage after indwelling of the Eluvia stent, and if that occurred, whether it was accompanied by endothelialization. Accumulation of cases and patho- logical evaluation are also necessary. The follow-up angiogram, IVUS and angioscope findings (Fig. 5, 6) suggested neointimal proliferation was well-controlled for at least one year, and neointimal coverage was completed within one year after Eluvia stenting. More follow-up data is needed to verify these findings.

\section{Limitation}

This study has certain limitations. First, it was a multicenter, prospective study, and detailed investigation of LLL was performed, but the sample size was relatively small. Regarding negative LLL and aneurysmal degeneration, further examination with a larger number of cases is necessary. Second, because we examined only a group of patients who consented to follow-up angiography, selection bias may be present. Third, in this study, the use of anticoagulants due to the history of atrial fibrillation was not significant difference. However, it was relatively higher based on the small sample size, and therefore might have influenced the chronic outcome. Much more data is needed to verify this finding. Lastly, IVUS and angioscope were performed only in some cases, and not all cases have been shown. However, we believe that the LLL results obtained in the present study are non-contradictory findings.

Despite these limitations, we suggest performing 

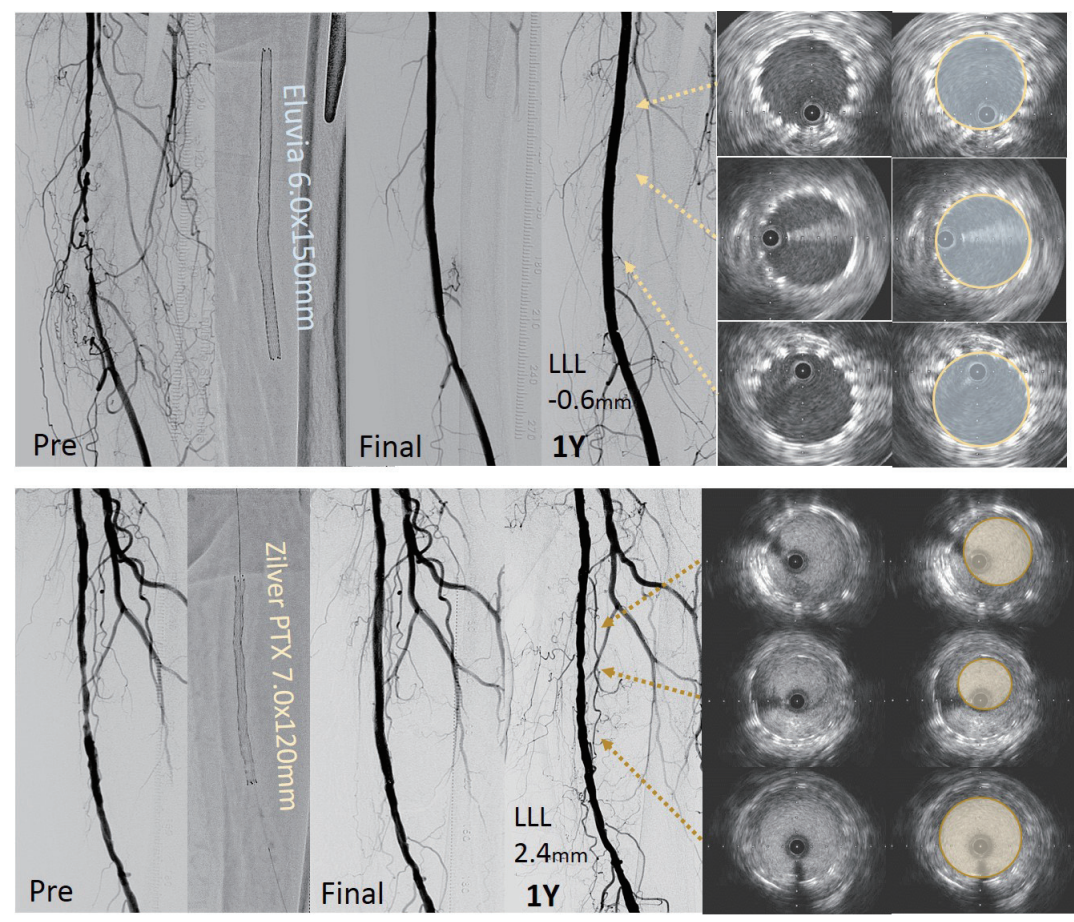

Fig. 5. Comparison of 1-Year IVUS Findings between Eluvia and Zilver PTX IVUS; intravascular ultrasound, LLL; late lumen loss
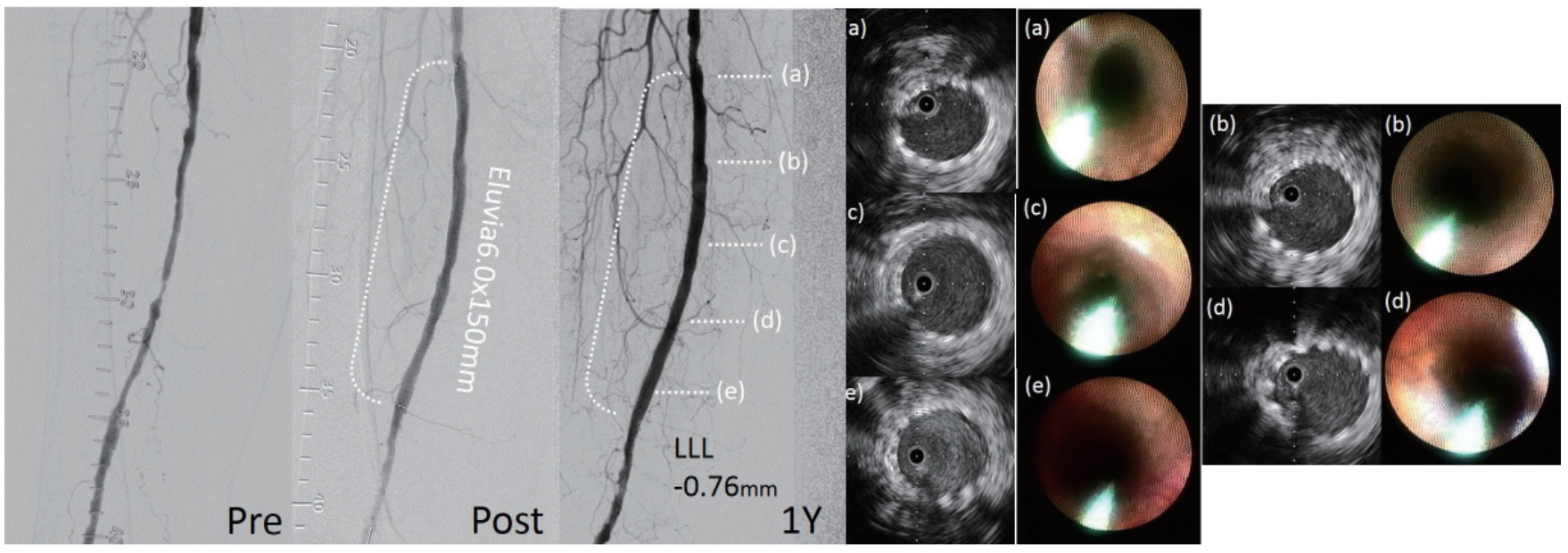

Fig. 6. Angioscope and IVUS Findings at 1-Year after Eluvia stenting

IVUS; intravascular ultrasound, LLL; late lumen loss From IVUS image, neointimal coverage was unclear after 1-year Eluvia placement. However, thin neointimal coverage without thrombus formation was found in angioscope.

a prospective, multicenter angiographic follow-up to consider the chronic phase outcome of DES.

\section{Conclusion}

Our study demonstrated that one-year LLL in the Eluvia group was significantly lower than that in the Zilver PTX group.

\section{Disclosure}

Yoshimitsu Soga, Masahiko Fujihara, Osamu Iida, and Daizo Kawasaki received honoraria (e. g. lecture fee) from the both Boston Scientific Japan and Cook Medical. 


\section{References}

1) Norgren L, Hiatt WR, Dormandy JA, Nehler MR, Harris KA, Fowkes FG; TASC II Working Group. Inter-society consensus for the management of peripheral arterial disease (TASC II). J Vasc Surg, 2007; 45: S5A-S67A

2) Kasapis C, Henke PK, Chetcuti SJ, Koenig GC, Rectenwald JE, Krishnamurthy VN, Grossman PM, Gurm HS. Routine stent implantation vs percutaneous transluminal angioplasty in femoropopliteal artery disease: a meta-analysis of randomized controlled trials. Eur Heart J, 2009; 30: 44-55

3) Schillinger M, Sabeti S, Loewe C, Dick P, Amighi J, Mlekusch W, Schlager O, Cejna M, Lammer J, Minar E. Balloon angioplasty versus implantation of nitinol stents in the superficial femoral artery. N Engl J Med, 2006; 354: 1879-1888

4) Krankenberg H, Schlüter M, Steinkamp HJ, Bürgelin K, Scheinert D, Schulte KL, Minar E, Peeters P, Bosiers M, Tepe G, Reimers B, Mahler F, Tübler T, Zeller T. Nitinol stent implantation versus percutaneous transluminal angioplasty in superficial femoral artery lesions up to $10 \mathrm{~cm}$ in length: the femoral artery stenting trial (FAST). Circulation, 2007; 116: 285-292

5) Bosiers M, Torsello G, Gissler HM, Ruef J, Müller-Hülsbeck S, Jahnke T, Peeters P, Daenens K, Lammer J, Schroë H, Mathias K, Koppensteiner R, Vermassen F, Scheinert D. Nitinol stent implantation in long superficial femoral artery lesions: 12-month results of DURABILITY I study. J Endovasc Ther, 2009; 16: 261-269

6) Laird JR, Katzen BT, Scheinert D, Lammer J, Carpenter J, Buchbinder M, Dave R, Ansel G, Lansky A, Cristea E, Collins TJ, Goldstein J, Jaff MR; RESILIENT Investigators. Nitinol stent implantation versus balloon angioplasty for lesions in the superficial femoral artery and proximal popliteal artery twelve-month results from the RESILIENT randomized trial. Circ Cardiovasc Interv, 2010; 3: 267-276

7) Laird JR, Schneider PA, Tepe G, Brodmann M, Zeller T, Metzger C, Krishnan P, Scheinert D, Micari A, Cohen DJ, Wang H, Hasenbank MS, Jaff MR; IN.PACT SFA Trial Investigators. Durability of Treatment Effect Using a Drug-Coated Balloon for Femoropopliteal Lesions: 24-Month Results of IN.PACT SFA. J Am Coll Cardiol, 2015; 66: 2329-2338

8) Rosenfield K, Jaff MR, White CJ, Rocha-Singh K, MenaHurtado C, Metzger DC, Brodmann M, Pilger E, Zeller T, Krishnan P, Gammon R, Müller-Hülsbeck S, Nehler MR, Benenati JF, Scheinert D; LEVANT 2 Investigators.
Trial of a Paclitaxel-Coated Balloon for Femoropopliteal Artery Disease. N Engl J Med, 2015; 373: 145-153

9) Müller-Hülsbeck S, Keirse K, Zeller T, Schroë H, DiazCartelle J. Long-Term Results from the MAJESTIC Trial of the Eluvia Paclitaxel-Eluting Stent for Femoropopliteal Treatment: 3-Year Follow-up. Cardiovasc Intervent Radiol, 2017; 40: 1832-1838

10) Gray WA, Keirse K, Soga Y, Benko A, Babaev A, Yokoi Y, Schroeder H, Prem JT, Holden A, Popma J, Jaff MR, Diaz-Cartelle J, Müller-Hülsbeck S; IMPERIAL investigators. A polymer-coated, paclitaxel-eluting stent (Eluvia) versus a polymer-free, paclitaxel-coated stent (Zilver PTX) for endovascular femoropopliteal intervention (IMPERIAL): a randomised, non-inferiority trial. Lancet, 2018; 392: 1541-1551

11) Rocha-Singh KJ, Zeller T, Jaff MR. Peripheral arterial calcification: prevalence, mechanism, detection, and clinical implications. Catheter Cardiovasc Interv, 2014; 83: E212220

12) Zhang YJ, Bourantas CV, Muramatsu T, Iqbal J, Farooq V, Diletti R, Campos CA, Onuma Y, Garcia-Garcia HM, Serruys PW. Comparison of acute gain and late lumen loss after PCI with bioresorbable vascular scaffolds versus everolimus-eluting stents: an exploratory observational study prior to a randomised trial. EuroIntervention, 2014; 10: 672-680

13) Iida O, Uematsu M, Soga Y, Hirano K, Suzuki K, Yokoi H, Muramatsu T, Inoue N, Nanto S, Nagata S. Timing of the restenosis following nitinol stenting in the superficial femoral artery and the factors associated with early and late restenoses. Catheter Cardiovasc Interv, 2011; 78: 611-617

14) Tsujimura T, Takahara M, Iida O, Hiramori S, Hayakawa N, Karashima E, Miura T, Teramura M, Ichihashi K, Kojima T, Aihara H, Yamaoka T, Fujihara M, Tosaka A, Doijiri T, Mano T, Soga Y. One-Year Clinical Outcomes following Implantation of InnovaTM Self-Expanding Nitinol Stents in Patients with Peripheral Artery Diseases Presenting Femoropopliteal Artery Lesions. J Atheroscler Thromb, 2019; 26: 847-855

15) Bisdas T, Beropoulis E, Argyriou A, Torsello G, Stavroulakis K. 1-Year All-Comers Analysis of the Eluvia DrugEluting Stent for Long Femoropopliteal Lesions After Suboptimal Angioplasty. JACC Cardiovasc Interv, 2018; 11: 957-966

16) Soga $Y$, Inoue K, Kuma S. Pathological findings of late stent thrombosis after paclitaxel-eluting stent implantation for superficial femoral artery disease. J Cardiol Cases, 2014; 11: 39-41 